\title{
Gastroenteritis de etiología viral en la era posvacunal frente a rotavirus: un estudio transversal retrospectivo de 5 años en un hospital al norte del Perù.
}

\author{
Franklin R. Aguilar-Gamboa 1,a, Lizzie K. Becerra-Gutierrez 2,b, Genesis M. Guevara-Vasquez ${ }^{3, c}$, \\ Katya Mariela Mera-Villasis ${ }^{1, d}$
}

\section{RESUMEN}

Objetivos. Determinar la frecuencia de casos de rotavirus y adenovirus en pacientes con sospecha de infección gastrointestinal de origen viral que asistieron a un hospital del norte del Perú, durante junio 2014 a noviembre 2018. Material y métodos: Se realizó un estudio transversal retrospectivo de 5 años. Para la detección, se emplearon pruebas de inmunocromatografía para la búsqueda simultánea de adenovirus y rotavirus en muestras heces. Resultados: El número total de pacientes fue de 64; de los cuales 13 $(20,31 \%)$ presentaron resultado positivo frente a rotavirus, y $4(6,25 \%)$ para adenovirus. Sin embargo, la proporción de casos negativos para ambos fue mayor. (73,44\%). Conclusión: A pesar de la gran efectividad de la vacuna frente a rotavirus, estos continúan siendo causa de gastroenteritis viral en la población infantil; Sin embargo, la proporción de casos negativos a los tests diagnósticos virales sugieren la presencia de otros agentes etiológicos.

Palabras clave: Rotavirus; norovirus; diarrea infantil (Fuente: DeCS-BIREME)

\section{Gastroenteritis of viral etiology in the post-vaccine era against rotavirus: a 5-year retrospective transversal study in a hospital north Peru.}

\section{ABSTRACT}

Objectives. to determine the frequency of cases of rotavirus and adenovirus in patients with suspected gastrointestinal infection of viral origin who attended a hospital in northern Peru during June 2014 to November 2018. Material and methods: A retrospective cross-sectional study of 5 years. For the detection, immunochromatography tests were used for the simultaneous search for adenovirus and rotavirus in stool samples Results The total number of patients was 64 ; of which $13(20.31 \%)$ were positive against rotavirus, and $4(6.25 \%)$ for adenovirus. However, the proportion of negative cases for both was higher. (73.44\%) Conclusión: Despite the great effectiveness of the rotavirus vaccine, these continue to cause viral gastroenteritis in children; However, the number of cases negative to viral diagnostic tests affected the presence of Other etiological agents.

Keywords: Rotavirus; norovirus; Diarrhea, Infantile (Source: MeSH-NLM)

\footnotetext{
${ }^{1}$ Laboratorio de Inmunología y Virologia, Hospital Regional de Lambayeque. Chiclayo, Perú. Grupo de investigación en Inmunología y Virología del Norte. Lambayeque, Perú.

2 Facultad de Medicina, Universidad de San Martin de Porres. Chiclayo, Perú. Laboratorio de Inmunología y Virología, Hospital Regional Lambayeque. Lambayeque, Perú.

${ }^{3}$ Dirección de investigación, Hospital Regional Lambayeque. Chiclayo, Perú. Evidence-Based Health Care South America: A Joanna Briggs Institute Affiliated Group, Universidad Norbert Wiener. Perú

a Biólogo, licenciado en Microbiología y Parasitología.

${ }^{\mathrm{b}}$ Doctora en Microbiología

- Licenciada en Estadística

${ }^{d}$ Maestra en Ciencias con mención en Microbiología
} 


\section{INTRODUCCIÓN}

La enfermedad diarreica aguda (EDA) representa la segunda enfermedad infecciosa más común en la población infantil (1) y es definida por la Organización Mundial de la Salud (OMS) como la disminución en la consistencia de las deposiciones con un aumento en el número de las mismas de 3 o más en $24 \mathrm{~h}$, que puede estar acompañada por fiebre o vómitos con una duración menor a 2 semanas. Estas infecciones son una causa importante de muertes en menores de cinco años con alrededor de 525000 decesos cada año, siendo además una de las principales causas de malnutrición de niños menores de cinco años ${ }^{(1)}$.

Los rotavirus del grupo A representa la principal causa de gastroenteritis aguda en bebés y niños pequeños en todo el mundo. De este modo se estima que a los 5 años de edad, casi todos los niños habrán sufrido al menos una infección por rotavirus ${ }^{(2)}$. Además, es probable que el mismo niño se infecte varias veces durante los primeros años de vida, aunque la sintomatología de la segunda infección suele ser más leve y la tercera suele ser asintomática ${ }^{(3)}$. En adultos, la infección por rotavirus suele ser asintomática o autolimitada, Sin embargo, puede ser grave en individuos inmunocomprometidos o en ancianos ${ }^{(4)}$.

Tanto los niños como los adultos infectados, eliminan los rotavirus en sus heces, lo que hace que el virus se propague fácilmente dentro de la comunidad. Por otro lado, los episodios de infección por rotavirus se asocian con una mayor gravedad clínica, mayores alteraciones en el comportamiento del niño y mayor angustia de los padres ${ }^{(5)}$.

Desde la introducción de la vacunación frente a rotavirus en el año 2006, se ha comprobado que la hospitalización por diarrea ha disminuido notablemente tanto en países desarrollados como en países en vías desarrollo ${ }^{(6)}$. Favorablemente, se ha observado una disminución de la tasa de hospitalización en niños mayores de cinco años, no vacunados, lo que implicaría la posible existencia de una protección indirecta o inmunidad tipo colectiva. Sin embargo, a la par con la disminución de los casos de rotavirus, otros agentes fueron tomando mayor protagonismo en el ámbito de la gastroenteritis de origen viral, es así que los casos ocasionados por norovirus empezaron a incrementar hasta llegar a ser en la actualidad una causa importante de gastroenteritis aguda viral en los seres humanos, caracterizada por producir brotes epidémicos en las comunidades, recintos militares, restaurantes, universidades, hospitales, bodas entre otras reuniones y eventos sociales ${ }^{(7)}$.

El centro de control de enfermedades (CDC) emitió en el 2009 el Sistema Nacional de Notificación de Brotes (NORS) para recolectar información sobre brotes de enfermedades transmitidas por los alimentos, por el agua y enfermedades entéricas que se propagan de persona a persona, a través de los animales o de las superficies en el ambiente. A través de este sistema disponible en Internet, las agencias de salud pública pueden reportar todos los brotes de gastroenteritis en Norteamérica, incluidas las enfermedades por rotavirus ${ }^{\left({ }^{8}\right)}$. Sin embargo, la organización de los datos y reportes de EDA viral en Latinoamérica aún tienen serias limitaciones, y aunque se incrementan los estudios y se adquieren tecnologías para su detección, el impacto de este tipo de infecciones todavía es a menudo subestimado. Por ello, la notificación y registro epidemiológico de las infecciones gastrointestinales son importantes para establecer en dónde ocurren los brotes, cómo se propagan estos virus y las fuentes alimentarias o acuáticas específicas que los causan. A su vez, esta información ayuda a identificar las mejores formas de prevenir y controlar este tipo de enfermedades. En este sentido, la presente tuvo como objetivo determinar la frecuencia de casos de rotavirus y adenovirus en pacientes con sospecha de EDA viral que asistieron a un hospital del norte del Perú durante junio 2014 a noviembre 2018.

\section{MÉTODOS}

Se realizó un estudio transversal retrospectivo en pacientes con sospecha de EDA viral que llegaron al laboratorio de inmunología y virología del Hospital Regional Lambayeque (HRL) durante el periodo de junio 2014 a noviembre 2018 para determinar la frecuencia de rotavirus y adenovirus en muestras de heces. La detección se realizó mediante test de inmunocromatografía de la marca RIDASCREEN® R-biopharm el cual permite la detección simultánea de adenovirus y rotavirus en heces. Se tomaron como criterios de inclusión a pacientes menores de 5 años procedentes del servicio de emergencia y pediatría del HRL, así como pacientes de la comunidad que fueron evaluados clínicamente y en los cuales se solicitó detección de etiología viral en heces. Se excluyeron de la evaluación los datos pertenecientes a muestras de heces que se informaron de tipo hemorrágicas o con cultivos bacterianos positivos y en los que se detectaron parásitos como Giardia intestinalis, Entamoeba histolytica/dispar entre otros. La EDA de etiología viral fue definida por un resultado positivo frente a detección de coproantígenos Vp6 y Hexón de rotavirus y adenovirus respectivamente presentes en heces sueltas o líquidas correspondiente al tipo 7 en la escala de Bristol.

\section{CONSIDERACIONES ÉTICAS}

El presente estudio fue evaluado y aceptado por el Comité de Ética en Investigación del Hospital Regional Lambayeque y registrado con el código 0211-005-19. 


\section{RESULTADOS}

El número total de pacientes fue de 64; de los cuales 13 $(20,31 \%)$ presentaron resultado positivo frente a rotavirus, 4 $(6,25 \%)$ a adenovirus y $47(73,44 \%)$ fueron negativos para ambos virus. No se presentaron casos de coinfecciones. El $59,4 \%(n=38)$ fueron de género masculino. La mayoría de los casos se presentaron entre enero y febrero de cada año, sin embargo, el mayor número de casos $(n=10)$ se presentó en octubre 2018. En cuanto a la prioridad de atención según la severidad de la infección 31 pacientes ingresaron por emergencia y 14 requirieron hospitalización, de los cuales se logró determinar etiología viral en el $32,3 \%(n=10)$ y el $28,6 \%(n=4)$ respectivamente. Aunque se detectó una mayor frecuencia de etiología por rotavirus que por adenovirus, la proporción de casos negativos para ambos fue mayor (Tabla 1). Finalmente, durante el periodo de estudio, se obtuvo en promedio 3 pacientes con resultados positivos independiente del tipo de virus, siendo los meses octubre $(n=10)$ y noviembre $(n=9)$ del año 2018 , los que presentaron los mayores casos positivos registrados (Gráfico 1).

Tabla 1. Características clínicas y demográficas de pacientes con sospecha de infección gastrointestinal de origen viral que asistieron al HRL durante junio 2014 a noviembre 2018.

\begin{tabular}{|c|c|c|c|}
\hline Variables & Rotavirus (\%) & Adenovirus (\%) & Negativo para ambos (\%) \\
\hline \multicolumn{4}{|l|}{ Edad } \\
\hline$<6$ meses & $3(18,8)$ & $1(6,3)$ & $12(75,0)$ \\
\hline de 6 meses a 1 año & 0 & $1(6,3)$ & $15(93,7)$ \\
\hline de 1 año a 2 años & $6(37,5)$ & 0 & $10(62,5)$ \\
\hline$>2$ años & $4(25,0)$ & $2(12,5)$ & $10(62,5)$ \\
\hline \multicolumn{4}{|l|}{ Sexo } \\
\hline Masculino & $7(18,4)$ & $2(5,3)$ & $29(76,3)$ \\
\hline Femenino & $6(23,1)$ & $2(7,7)$ & $18(69,2)$ \\
\hline \multicolumn{4}{|l|}{ Procedencia } \\
\hline Consulta Externa & $3(17,4)$ & 0 & $14(82,6)$ \\
\hline Emergencia & $7(22,6)$ & $3(9,7)$ & $21(67,7)$ \\
\hline Hospitalización Pediatría & $3(21,4)$ & $1(7,2)$ & $10(71,4)$ \\
\hline Áreas Críticas & 0 & 0 & $2(100,0)$ \\
\hline \multicolumn{4}{|l|}{ Hallazgo etiológico } \\
\hline Negativo & 0 & 0 & $47(100,0)$ \\
\hline Positivo & $13(76,5)$ & $4(23,5)$ & 0 \\
\hline
\end{tabular}

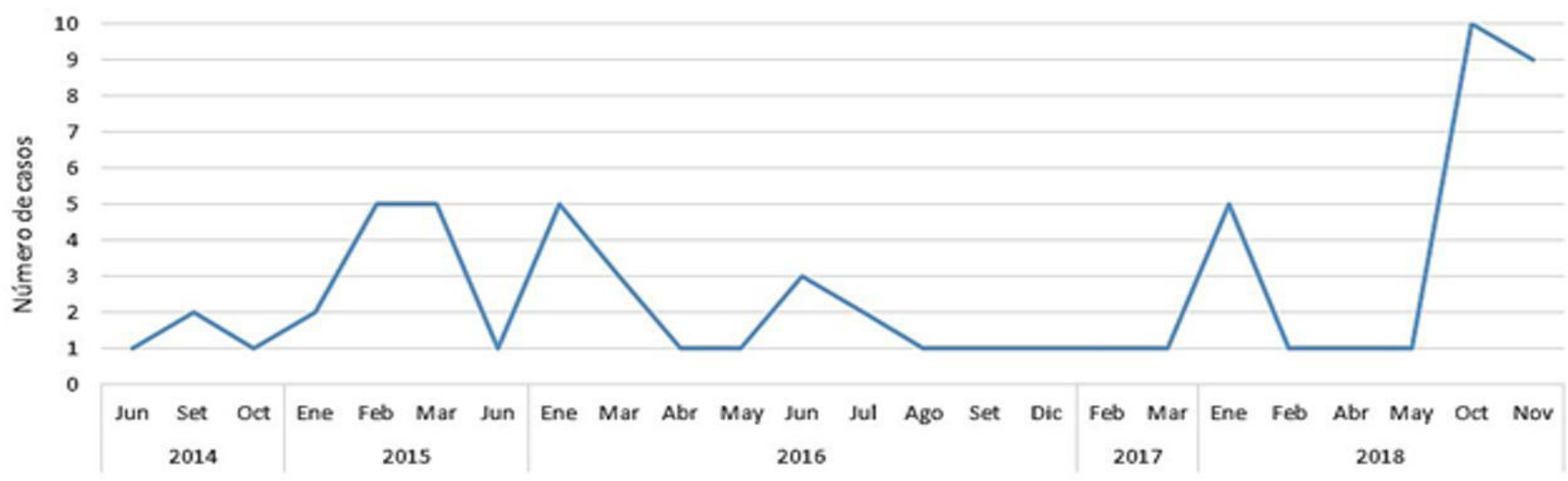

Periodo de Atención según mes

Gráfico 1: Frecuencia de casos con sospecha de infección gastrointestinal de origen viral en pacientes que asistieron al HRL según mes, durante junio 2014 a noviembre 2018. 


\section{DISCUSIÓN}

El manejo de la EDA es principalmente clínico. Este puede consistir en rehidratación y reposición de electrolitos o en algunos casos el uso de antimicrobianos como en las diarreas invasivas. De este modo, son pocas las ocasiones en las que se necesita una evaluación posterior con resultados de coprocultivos o pruebas de detección de agentes virales en heces. Por ello, los pocos datos regionales con que contamos al respecto proceden de registros epidemiológicos y de trabajos de investigación.

Estudios previos en la región Lambayeque, al norte de Perú, describen una frecuencia de $5,8 \%$ de etiología viral en pacientes que asistieron al HRL con sospecha de EDA en 2015. Por otro lado, informaron que el $51,4 \%$ de muestras no presentaron agente etiológico identificable ${ }^{(9)}$. Esto se reafirma con lo hallado en nuestro estudio, ya que dentro de los casos de EDA con sospecha viral que se atendieron en el $\mathrm{HRL}$, se tuvo un $73,44 \%$ de casos que se informaron como negativos. Este hecho llama la atención debido a la probabilidad de que exista todavía un amplio grupo de agentes infecciosos que no se estén considerando en la búsqueda como causantes de EDA en esta región.

Por otro lado, es importante mencionar que durante los 5 años en el que se recopilaron los datos para el presente estudio, tan solo se realizaron 64 solicitudes para investigar EDA de origen viral. Esto revela que el manejo empírico prevalece en este tipo de infecciones y que se otorga poca importancia a la búsqueda del agente etiológico específico en nuestro medio, hecho que contrasta con la situación de países desarrollados ${ }^{(10)}$.

En cuanto a la etiología viral hallada en el presente estudio el $76,5 \%$ de los casos positivos fueron causados por rotavirus mientras que el 23,5 restante lo fue por adenovirus. A pesar de ello, el número de casos negativos fue considerablemente mayor (ver tabla 1). Esto revela que aunque aún se presentan casos producidos por ambos virus, su frecuencia es reducida en nuestro medio, esto debido principalmente a la eficacia de la vacuna frente a rotavirus la cual desde su instauración en el año 2006 demuestra un buen perfil de seguridad, y ofrece protección amplia a los serotipos circulantes, con una inmunogenicidad moderada $\lg \mathrm{A}$ anti rotavirus en suero y una baja excreción viral (11). Sin embargo, el número de casos negativos en las muestras diarreicas con alta sospecha de etiología viral nos hacen sugerir poderosamente la presencia de otros agentes que no se estarían considerando. Así mismo, para el análisis e interpretación de los resultados obtenidos es importante mencionar el desempeño del test de inmunocromatografia RIDASCREEN $®$ R-biopharm, el cual en estudios comparativos tomando como patrón a pruebas moleculares demostraron una excelente sensibilidad $(100 \%)$ pero una baja especificidad $(72 \%)^{(12)}$.
A pesar de la instauración de la vacuna frente a rotavirus, en algunos países del mundo la diarrea es una causa importante de morbilidad y mortalidad en los niños. En países como Nigeria, los casos en menores de 5 años se detectan hasta en un $55,9 \%{ }^{(13)}$. Estos datos que contrastan con nuestros hallazgos, se explicarían debido a las características socioeconómicas de cada país y en sus políticas de aplicación de esquemas de vacunación. Aunque también debemos considerar la efectividad de estas vacunas frente a distintos genotipos de rotavirus circulantes y la presión selectiva que pueden ejercer las mismas, un hecho observado con cepas de G2P, después de la introducción de la vacuna monovalente contra rotavirus G1P en varios países de América del Sur, Europa, y Australia. Así mismo, también se han observado aumentos similares en la prevalen-cia de las cepas G2P en países que introdujeron la vacuna contra el rotavirus pentavalente (que incluye el genotipo G2) y en países con bajo uso de la vacuna contra el rotavirus. Por lo tanto, no está claro si las diferencias en los genotipos de rotavirus representan la variación estacional natural o la presión de selección inducida por la vacunación. Estos hallazgos enfatizan la necesidad de vigilancia continua para monitorear los cambios en la epidemiología del rotavirus luego de la intro-ducción de la vacuna ${ }^{(14)}$.

En algunos estudios se informa que la tasa más alta de gastroenteritis viral se encuentra en pacientes de género masculino ${ }^{(15)}$. Sin embargo en nuestro estudio esta diferencia fue insignificante. Por otro lado el mayor número de casos ingresaron por el servicio de emergencia y solo 2 pacientes con sospecha de EDA de origen viral que presentaron resultados negativos a rotavirus y adenovirus requirieron ingreso a áreas críticas y si consideramos que para el presente estudio se excluyeron aquellos pacientes con sospecha de una etiología distinta a la viral. Esto último sugeriría la presencia de otros agentes como norovirus, los cuales podrían ser los responsables de ocasionar estos cuadros complicados en pacientes pediátricos.

En cuanto a la temporalidad de los hallazgos, se describe que los casos se presentaron entre enero y febrero de cada año con un pico en octubre 2018. Lo cual contrasta con el estudio de Akan et. Al 2009 realizado en Turquía quienes determinaron que la mayoría de los casos positivos de rotavirus se observaron en diciembre, enero, febrero y marzo $(p<0,001)$. Meses que se caracterizan por temporal frío en este país ${ }^{(16)}$. $Y$ aunque es difícil realizar una adecuada comparación debido a las características demográficas y climatológicas distintas de cada lugar, es posible determinar un patrón estacional que acompaña a la detección de estos virus en los casos de diarrea. 
Rotavirus y adenovirus son causas importantes de diarrea viral en muchos países y han provocado un número considerable de muertes en niños en la última década. Sin embargo, en la actualidad otros virus, como los norovirus y los astrovirus, representan la mayoría de las infecciones virales por gastroenteritis documentadas en algunos países desarrollados ${ }^{(17)}$. De sugerir la presencia de otros agentes virales en los casos negativos obtenidos en nuestro estudio, deberíamos considerar notablemente la sospecha de norovirus. Este agente infeccioso presenta ventajas evolutivas frente a otros virus productores de EDAs, por ejemplo la evasión de las respuestas inmunitarias del huésped mediante la deriva antigénica, a través de un proceso similar al del virus de la gripe, su capacidad de resistir condiciones extremas, el no solo ser frecuente en infantes y su persistencia y contaminación por fómites hacen que se requieran medidas como el lavado de manos obligatorio (debido a la ineficacia de los desinfectantes tópicos), desinfección de superficies y lavado de ropa, como medida de contención y prevención de la transmisión de este virus altamente contagioso.

Una de las consecuencias del desconocimiento de la etiología de origen viral, es el inicio de tratamiento antimicrobiano empírico en casos de EDA, el cual no solo contribuye al desarrollo de resistencia antimicrobiana sino a la alteración de la microbiota intestinal lo cual puede contribuir a elevar la morbilidad en la infección. Si bien el manejo empírico es fundamental y necesario en estos casos, no podemos subestimar la identificación del agente etiológico y confiar tan sólo en parámetros clínicos. En este sentido se conoce que la presencia de leucocitos es usualmente tomado como un indicador de infección bacteriana, sin embargo estudios realizados al respecto, revelan que los ca-sos positivos para rotavirus pueden tener una prueba de leucocitos fecales positiva hasta en un $46.9 \%{ }^{(8)}$; es decir casi la mitad de los casos, y aunque la probabilidad de encontrar positiva esta prueba fue menor cuando la presencia de leucocitos fecales fue fuertemente positiva (6.3\%), es evidente que la reacción inflamatoria posee mayor utilidad para la determinación de un proceso infeccioso bacteriano que viral, y aunque un estudio coprológico funcional podría otorgarnos mayores datos para esta determinación, su solicitud es tan igual o menor que la determinación de agen-tes virales en heces.

Este estudio presenta algunas limitaciones: a) No se realizó la detección de norovirus astrovirus y sapovirus en los casos estudiados debido a que estos no son considerados en las pruebas de rutina diagnóstica. Lo cual, ante los resultados obtenidos, tal vez pueda ser un factor a ser reconsiderado. b) No se investigó si los niños presentaban vacunación previa frente a rotavirus, lo cual aportaría importante información sobre la efectividad de la vacuna y la tasa de reinfección. c) No se investigó las características clínico epidemiológicas como condiciones socioeconómicas, acceso a servicios públicos de saneamiento, entre otros.

Se concluye que desde la introducción de la vacuna frente a rotavirus en 2006, los casos graves, hospitalizaciones y mortalidad han disminuido considerablemente en el mundo. Sin embargo, estos virus continúan siendo causa importante de gastroenteritis en la población infantil. La proporción de casos negativos a los tests diagnósticos virales sugieren la presencia de otros agentes etiológicos causantes de diarrea, en este sentido otros virus enteropatógenos deben ser considerados en los estudios de rutina principalmente norovirus el cual es ampliamente reconocido como un virus emergente que requiere atención.

Fuente de financiamiento: Autofinanciado.

Conflicto de interéses: Los autores no poseen conflicto de interés.

\section{REFERENCIAS BIBLIOGRÁFICAS}

1. World Health Organization. Enfermedades diarreicas [Internet]. WHO 2017 [cited 2019 Feb 21]. Available from: https://www.who.int/es/ news-room/fact-sheets/detail/diarrhoeal-disease

2. Rotavirus vaccines WHO position paper: January 2013 - Recommendations. Vol. 31, Vaccine. 2013. p. 6170-1.

3. Velázquez FR. Protective effects of natural rotavirus infection. Pediatr Infect Dis J [Internet]. 2009 Mar [cited 2020 Mar 5];28(SUPPL. 3):S546. Available from: http://www.ncbi.nlm.nih.gov/pubmed/19252424

4. Anderson EJ, Weber SG. Rotavirus infection in adults. Vol. 4, Lancet Infectious Diseases. Lancet Publishing Group; 2004. p. 91-9.

5. Arístegui J, Ferrer J, Salamanca I, Garrote E, Partidas A, San-Martin $M$, et al. Multicenter prospective study on the burden of rotavirus gastroenteritis in children less than 3 years of age in Spain. BMC Infect Dis [Internet]. 2016 Oct 10 [cited 2020 Mar 5];16(1):549. Available from: http://www.ncbi.nlm.nih.gov/pubmed/27724892

6. World Health Organization. WHO | Global Rotavirus Information and Surveillance Bulletin [Internet]. WHO. World Health Organization; 2014 [cited 2019 Feb 21]. Available from: https://www.who.int/immunization/diseases/rotavirus/rota_info_surv_bulletin/en/

7. Hardstaff JL, Clough HE, Lutje V, Mclntyre KM, Harris JP, Garner P, et al. Foodborne and Food-Handler Norovirus Outbreaks: A Systematic Review. Foodborne Pathog Dis [Internet]. 2018 [cited 2019 Mar 20];15(10):589-97. Available from: http://www.ncbi.nlm.nih.gov/pub$\mathrm{med} / 30109958$

8. National Center for Immunization and Respiratory Diseases D of VD. Norovirus Trends and Outbreaks | CDC [Internet]. June 1. 2018 [cited 2019 Jan 26]. Available from: https://www.cdc.gov/norovirus/ trends-outbreaks/index.html

9. Silva-Díaz H, Bustamante-Canelo O, Aguilar-Gamboazsu F-R, Mera-Villasis K, Ipanaque-Chozo J, Seclen-Bernabe E, et al. Enteropatógenos predominantes en diarreas agudas y variables asociadas en niños atendidos en el Hospital Regional Lambayeque, Perú. Revista Horizonte Médico. [Internet]. Vol. 17, Horizonte Médico. Universidad de San Martín de Porres. Facultad de Medicina Humana; 2017 [cited 2019 Feb 22]. 38-44 p. Available from: http://www.scielo.org.pe/scielo.php?script=sci_arttext\&pid=S1727-558X2017000100007

10. Chen C, Wang LP, Yu JX, Chen X, Wang RN, Yang XZ, et al. Prevalence of enteropathogens in outpatients with acute diarrhea from urban and rural Areas, Southeast China, 2010-2014 [Internet]. Vol. 101, American Journal of Tropical Medicine and Hygiene. American Society of Tropical Medicine and Hygiene; 2019 [cited 2020 Mar 5]. p. 310-8. Available from: http://www.ncbi.nlm.nih.gov/pubmed/31219001 
11. Blanco NA, Castillo SF. Vacunas contra rotavirus: Estado actual y tendencias futuras. Vaccimonitor. 2016;25(3):89-97.

12. Sukran A, Altay A, Selma G, Mehmet A, Nurgul B, Nuri C, et al. Investigation of Rotavirus with Various Methods in Children with Acute Gastroenteritis and Determination of Its Molecular Epidemiology in Kayseri Province, Turkey. J. Clin. Lab. Anal. 2017; 31:1-5.

13. Odimayo MS, Olanrewaju WI, Omilabu SA, Adegboro B. Prevalence of rotavirus-induced diarrhoea among children under 5 years in llorin, Nigeria. J Trop Pediatr. 2008;54(5):343-6.

14. Crawford SE, Ramani S, Tate JE, Parashar UD, Svensson L, Hagbom M, et al. Rotavirus infection. Nat Rev Dis Prim. 2017 Nov 9;3:17083.

15. Topkaya A, Aksungar B, Özakkafl F. Examination of rotavirus and enteric adenovirus in children with acute gastroenteritis. Türk Mikrobiyol Cem [Internet]. 2006;36(March 2005):210-3. Available from: http://www.alka. com.br/site/files/upload/1074.pdf

16. Akan H, İzbırak G, Gürol Y, Sarıkaya S, Gündüz TS, Yılmaz G, et al. Rotavirus and adenovirus frequency among patients with acute gastroenteritis and their relationship to clinical parameters: a retrospective study in Turkey. Asia Pac Fam Med. 2009;8(1):8.

17. Tran A, Talmud D, Lejeune B, Jovenin N, Renois F, Payan C, et al. Prevalence of rotavirus, adenovirus, norovirus, and astrovirus infections and coinfections among hospitalized children in Northern France. J Clin Microbiol. 2010 May 19;48(5):1943-6. 